\title{
Distribution of the D15Z1 copy number polymorphism
}

\author{
Annette E Cockwell ${ }^{*}$, Patricia A Jacobs ${ }^{1,2}$ and John A Crolla ${ }^{1,2}$ \\ ${ }^{1}$ Wessex Regional Genetics Laboratory, Salisbury District Hospital, Salisbury, Wiltshire, SP2 8BJ, UK; ${ }^{2}$ Division of \\ Human Genetics, Southampton General Hospital, Southampton, UK
}

Using fluorescent in situ hybridization (FISH) with the probe p15 (D15Z1), we investigated the distribution of the polymorphic $15 p$ signal which has been reported to occur on acrocentric chromosomes in addition to chromosome 15. The short arm of chromosome 15 has a characteristic signal pattern when hybridized with the FISH probe D15Z1. However, the D15Z1 signal can occasionally be seen on the short arm of other acrocentric chromosomes. We studied the distribution of the $\mathrm{D} 15 \mathrm{Z1}$ probe in 1657 patients consisting both of individuals with a normal karyotype and those with a variety of chromosome abnormalities involving the acrocentric chromosomes. Our results show that one in six individuals, regardless of their patient ascertainment category or karyotypic status, had one or more additional D15Z1 signals, and that there were no significant differences in the distribution of extra signals among the patient groups. European Journal of Human Genetics (2007) 15, 441-445. doi:10.1038/sj.ejhg.5201780; published online 21 February 2007

Keywords: D15Z1; copy number; 15p polymorphism

\section{Introduction}

A staining technique using distamycin A/4',6-diamino-2phenyl-indole (DA/DAPI) was devised in the late 1970s that was specific for the heterochromatic regions of chromosomes 1, 9, 16, Y and the short arms (band p11) of chromosome $15 .{ }^{1}$ Its initial clinical cytogenetic use was for identifying whether or not bisatellited supernumerary marker chromosomes were derived from chromosome 15. Reports were subsequently published describing staining intensity polymorphisms of the chromosome 15 short $\operatorname{arms}^{2}$ and of DA/DAPI signals being occasionally present on acrocentric chromosomes other than chromosome $15 .^{3}$

In situ hybridization using D15Z1, a probe that is specific for the short arm of chromosome 15, superseded DA/DAPI staining. D15Z1 was first isolated by Higgins $e t \mathrm{al}^{4}$ and is a repetitive $1.8 \mathrm{~kb}$ Kpnl DNA sequence which under condi-

${ }^{*}$ Correspondence: Annette E Cockwell, Wessex Regional Genetics Laboratory, Salisbury District Hospital, Salisbury, Wiltshire, SP2 8B], UK. Tel: + 441722336262 ext 4080; Fax: + 441722 338095;

E-mail: annette.cockwell@salisbury.nhs.uk

Received 20 September 2006; revised 13 December 2006; accepted 20 December 2006; published online 21 February 2007 tions of high stringency hybridizes specifically to the chromosome 15 short arm (15p11). It consists of tandemly arranged imperfect repeats of $5^{\prime}$ AATGG $3^{\prime}$ similar to other satellite III sequences, and comprises the major part of the heterochromatin of the chromosome 15 short arm. D15Z1 is used for supernumerary marker chromosome identification and Y;15 translocations ${ }^{5}$ as well as being an indicator of chromosome 15 in studies involving duplications and deletions of the chromosome 15 long arm such as PraderWilli and Angelman cases. ${ }^{6}$

In 1991, Smeets et $\mathrm{al}^{7}$ using the DA/DAPI method examined 127 patients, referred for cytogenetic analysis, and found that seven had three DA/DAPI positive acrocentric chromosomes; the additional signals were on chromosome 14 in six cases, and on a chromosome 13 in the other. Subsequently, Stergianou et $a l^{8,9}$ using in situ hybridization D15Z1 investigated 100 patients and found 12 to have signals on three acrocentric chromosomes, all the extra signals being on chromosome 14; moreover in four families the additional D15Z1 signal was shown to be inherited. A few years later, Shim et al ${ }^{6}$ examined 109 patients, referred because of a suspected structural rearrangement of the long arm of chromosome 15 (eg Prader-Willi/ 
Angelman syndromes, autism studies), and found nine with an extra signal on chromosome 14, one patient having both chromosome 14s positive for D15Z1 and one patient with only one chromosome 15 signal.

We present a study of 1657 patients, who we examined as part of ongoing studies of chromosome abnormalities involving the pericentromeric regions of the acrocentric chromosomes with particular focus on couples ascertained because of three or more pregnancy losses. ${ }^{10}$ We used fluorescent in situ hybridization (FISH) with D15Z1, to investigate the distribution of the $15 \mathrm{p} 11$ polymorphism amongst four different and distinct groups of patients. We wished to determine, firstly, if the occurrence of the $15 p$ polymorphism varied in different populations of cytogenetically normal patients and secondly, to determine the frequency of the $15 \mathrm{p}$ polymorphism in individuals with an abnormality of one or more acrocentric chromosomes. This would enable us to determine whether the $15 \mathrm{p}$ polymorphism played a role in the genesis of chromosome abnormalities involving the acrocentric chromosomes or whether it was an apparently harmless polymorphism.

\section{Materials and methods Study population}

The study population consists of 1657 patients divided into four groups. Firstly, three groups with normal chromosomes using conventional cytogenetics (ISCN 550 bands) namely (i) A group of 636 patients referred following recurrent miscarriages, (ii) A group of 200 control patients who are unaffected relatives in families in which one or more individuals have a structural chromosome rearrangement and (iii) A group of 500 children referred for cytogenetic testing. Secondly, (iv) a group of 322 patients who have an abnormality involving one or more acrocentric chromosomes (see Table 1).

\section{FISH}

The chromosome 15 short arm probe p15 (D15Z1) and the 15 centromere specific probe pTRA-25 (D15Z3) were used to screen for the $15 p$ polymorphisms, the latter was used to facilitate the identification of the chromosome 15 homologues. Probe DNA was extracted from glycerol stocks using rapid alkaline lysis ${ }^{11}$ and labelled using a nick translation reaction with biotin-16-dUTP for the 15 centromere probe (pTRA-25) and digoxigenin-11-dUTP (Boehringer-Mannheim, UK) for the 15 short arm probe (p15).

The FISH experiments were based on a modification of the technique of Pinkel et al. ${ }^{12}$ The slides were examined using a Pinkel Fluorescent no. 83 filter series (Chroma Technology) fitted to a Carl Zeiss Axiophot epifluorescence microscope with a $50 \mathrm{~W}$ mercury vapour UV light source. A minimum of three metaphases from each patient were
Table 1 Summary of additional $15 p$ signals by patient group

\begin{tabular}{|c|c|c|c|}
\hline \multirow[b]{2}{*}{ Patient group } & \multirow[b]{2}{*}{ No. patients } & \multicolumn{2}{|c|}{$\begin{array}{l}\text { Patients with additional } \\
15 p \text { signal }\end{array}$} \\
\hline & & Total & $\%$ \\
\hline $\begin{array}{l}\text { Recurrent miscarriage } \\
\text { Relatives controls }^{\mathrm{b}} \\
\text { Children controls }^{\mathrm{c}}\end{array}$ & $\begin{array}{l}636 \\
200 \\
500\end{array}$ & $\begin{array}{r}118 \\
32 \\
92\end{array}$ & $\begin{array}{l}18.5 \\
16.0 \\
18.4\end{array}$ \\
\hline Total normal karyotypes & 1336 & 242 & 18.1 \\
\hline $\begin{array}{l}\text { Acrocentric trisomies } \\
+13 \\
+15 \\
+21 \\
+22\end{array}$ & $\begin{array}{r}18 \\
1 \\
200^{d} \\
1\end{array}$ & $\begin{array}{r}3 \\
32\end{array}$ & \\
\hline Total trisomies & 220 & 35 & 15.9 \\
\hline $\begin{array}{l}\text { Supernumerary markers } \\
13 / 21 \\
14 \\
15 \\
22\end{array}$ & $\begin{array}{r}2 \\
1 \\
45 \\
12\end{array}$ & $\begin{array}{l}7 \\
4\end{array}$ & \\
\hline Total markers & 60 & 11 & 18.5 \\
\hline $\begin{array}{l}\text { Robertsonian translocations } \\
13 ; 13 \text { unbal }(+13) \\
13 ; 14 \\
13 ; 14 \text { unbal }(+13) \\
13 ; 15 \\
13 ; 21 \text { unbal }(+21) \\
13 ; 22 \\
14 ; 15 \\
14 ; 21 \\
14 ; 22 \\
21 ; 21 \text { unbal }(+21)\end{array}$ & $\begin{array}{r}3 \\
25 \\
1 \\
1 \\
1 \\
1 \\
4 \\
3 \\
1 \\
2\end{array}$ & $\begin{array}{l}1 \\
3\end{array}$ & \\
\hline Total Robertsonians & 42 & 4 & 9.5 \\
\hline $\begin{array}{l}\text { Total acrocentric } \\
\text { abnormalities }\end{array}$ & 322 & 50 & 15.5 \\
\hline Total & 1657 & 292 & 17.6 \\
\hline
\end{tabular}

aKnown to have had three or more pregnancy losses.

${ }^{b}$ Relatives (mostly parents) of probands who had a structural chromosome abnormality (excluding probands referred with reproductive problems).

${ }^{c}$ Referred either for fragile $X$ testing, developmental delay, dysmorphism or failure to thrive.

${ }^{\mathrm{d}}$ High number reflecting the frequency of this trisomy in diagnostic cytogenetic laboratories.

examined by eye, but in addition, images were visualized and captured using a cooled charged-coupled device camera with Perceptive Scientific International (PSI) MacProbe software. A signal for D15Z1 was deemed to be present if it was consistently seen as a clear signal in interphase cells as well as the metaphases. 


\section{Results}

Under the experimental conditions used, FISH with the probe D15Z1 showed a unique signal on the chromosome 15 short arm in the majority of cells examined (Figure 1a). The centromere probe, pTRA-25, used to identify the chromosome $15 \mathrm{~s}$, showed that the 15 centromere alphoid sequences had no involvement with the additional D15Z1 signals observed on the other acrocentrics. Overall, 18\% of the patients studied had a signal on an acrocentric chromosome in addition to the chromosome $15 \mathrm{~s}$ and these additional signals could also be resolved in the interphase cells (Figure 1b). Furthermore, the proportion was remarkably consistent among all the groups studies (Table 1) and between males and females (data not shown).
The additional D15Z1 signals were seen on all the acrocentric chromosomes, but their distribution among the different autosomes varied dramatically (Table 2). Approximately $60 \%$ were on chromosome $14,23 \%$ on chromosome 13 , whereas the remainder were distributed between chromosomes 21, 22 and cells with more than one additional chromosome 15 polymorphism (Table 2 ).

Morphologically, the D15Z1 signal seen on chromosome 14 sometimes appeared as large as that on the 15 homologues, but often appeared smaller, whereas, those hybridized to chromosome 13 were invariably smaller (Figure 1c), although still visible in the interphase cells. When the extra D15Z1 signal was seen on chromosomes 21 or 22 , its size was more variable, ranging from very small to
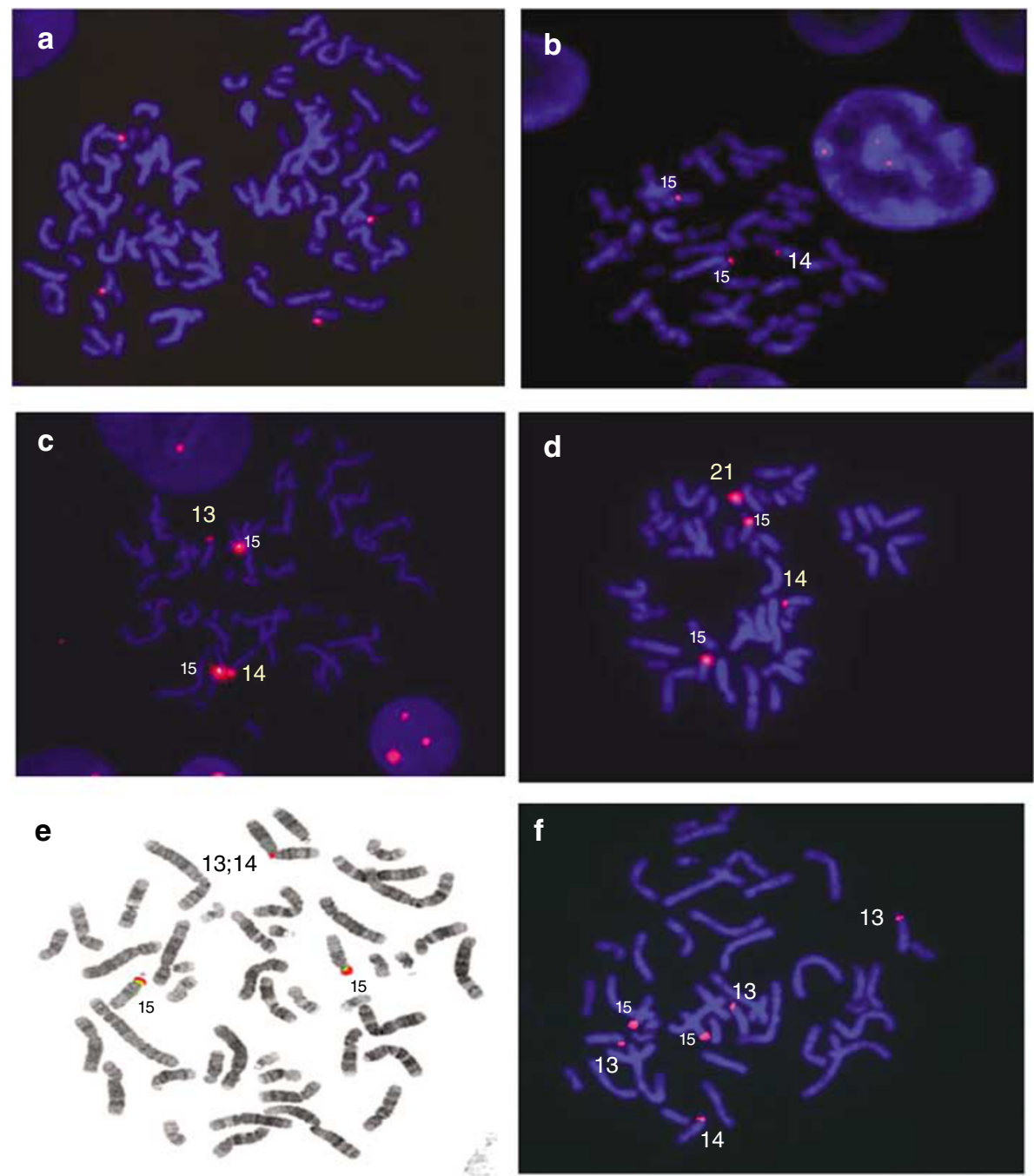

Figure 1 (a) Two cells showing D15Z1 hybridizing specifically to the short arms of chromosome 15. (b) D15Z1 hybridized to the short arm of a chromosome 14 with a corresponding additional signal an interphase cell. (c) The additional signal on chromosome 14 is nearly as large as that on chromosome 15, whereas the chromosome 13 signal is a pinprick. (d) The signal on chromosome 21 is larger than those on chromosome 15 or the pinprick on chromosome 14. (e) D15Z1 signal in the gap between the centromeres in a 13;14 Robertsonian dicentric chromosome. (f) Trisomy 13 with all three chromosome $13 \mathrm{~s}$, one chromosome 14 and two chromosome $15 \mathrm{~s}$ with D15Z1 signals. 
Table 2 Summary of chromosomes with $15 p$ polymorphisms

\begin{tabular}{|c|c|c|c|c|c|c|c|c|c|c|c|}
\hline & \multicolumn{5}{|c|}{ Total no. patients Total with extra D15Z1 } & \multicolumn{6}{|c|}{ Chromosome(s) with $15 p$ polymorphism } \\
\hline $\begin{array}{l}\text { No. } \\
\text { \% with extra D15Z1 }\end{array}$ & 1657 & 292 & $\begin{array}{l}13 \\
66 \\
22.6\end{array}$ & $\begin{array}{c}14 \\
174 \\
59.59\end{array}$ & $\begin{array}{c}21 \\
14 \\
4.8\end{array}$ & $\begin{array}{l}22 \\
7 \\
2.4\end{array}$ & $\begin{array}{r}13+14 \\
11 \\
2.77\end{array}$ & $\begin{aligned} 14 \times 2 \\
6 \\
2.05\end{aligned}$ & $\begin{array}{r}14+21 \\
3 \\
1.03\end{array}$ & $\begin{array}{r}14+22 \\
6 \\
2.05\end{array}$ & $\begin{array}{l}\text { Others } \\
5 \\
1.71\end{array}$ \\
\hline
\end{tabular}

larger that that seen on the chromosome 15s (Figure 1d). As far as we are aware, there is no precedent for these observations.

Although in the great majority of patients the additional D15Z1 signal was located on the proximal short arm (band p11) of an acrocentric chromosome, there were a number of exceptions. In nine cases, the extra D15Z1 signal was observed on the satellites (band p13); in two cases it was seen on the chromosome 15 satellites as well as p11. One of the patients with a balanced 13;14 Robertsonian translocation had a D15Z1 signal present in the gap between the centromeres in the dicentric (Figure 1e) which suggests that the D15Z1 event happened before the Robertsonian translocation was formed. One trisomy 13 patient had all three chromosomes 13 and one chromosome 14 with signals, which along with the chromosome $15 \mathrm{~s}$ made a total of six D15Z1 signals per cell (Figure 1f); unfortunately the parents were not available, so the origin of the chromosomes with the additional signals could not be determined.

Two patients were apparently hemizygous for a D15Z1 signal, which was also observed in one patient from Shim et al's $^{6}$ study. One of our patients had apparently normal chromosome $15 \mathrm{~s}$ by conventional cytogenetics, but FISH showed that one of the chromosome 15s had, in fact, a chromosome 14 centromere and presumably 14 short arms $(\operatorname{der}(14) \mathrm{t}(14 ; 15))$. A further patient had a chromosome 15 with a 15 centromere, but the short arm did not hybridize with D15Z1 or probes for Yqh (pHY2.1, Cooke, Imperial Cancer Research Fund), 9qh (pHuR98, American Type Culture Collection) or 16qh (pHuR195, American Type Culture Collection) and so it was presumed that the short arm was derived from one of the other acrocentric chromosomes.

In four families, where parents were available to study the inheritance of the additional D15Z1 signal, three probands inherited the additional signal from their mother and one from their father.

\section{Discussion}

The results presented in the present study of 1657 patients show that overall 292 have an additional D15Z1 signal on acrocentric chromosomes other than chromosome 15 . Consequently as shown by this and other studies, despite giving a good hybridization signal where present, the propensity of D15Z1 to be found on chromosomes other than 15 , precludes it from being the probe of choice when distinguishing chromosome 15 from other acrocentric chromosomes, particularly if only interphase cells are available. In our study, the majority of the additional signals were on chromosome 14 ( $\sim 1$ in 9.5 individuals), but in contrast with previous studies a proportion of patients were found to have the additional signal on chromosome 13 ( $\sim 1$ in 26$)$, chromosome 21 ( $\sim 1$ in 118) and chromosome $22(\sim 1$ in 236$)$, as well as in exceptional cases, combinations of multiple signals. It is now known that copy number variation of other DNA homologous sequences can be complex and involve gains (as well as losses) at more than one site in the genome and this also seems to be the case with the chromosome 15 short arm. ${ }^{13}$

Given the patients with additional $15 \mathrm{p}$ signals and assuming Mendelian inheritance, the number where both chromosome 14 s had a D15Z1 signal $(n=6)$ is as expected $(\sim 5)$. Nevertheless, it is still an uncommon enough finding for uniparental disomy for chromosome 14 to be considered. ${ }^{6}$ However, given the single chromosome signal frequencies, all the other multiple signal combinations were seen between 6-33 times more often than expected.

In conclusion, we have studied 1657 patients with a range of referral reasons. We found that there was no significant difference in the distribution of additional signals among the patient groups. In general, the size of the additional $15 \mathrm{p}$ signal is different depending on the acrocentric chromosome it hybridizes to, but the reason for this is not obvious. As the frequency is not increased in patients with chromosome abnormalities, it does not appear to play a role in the genesis of such abnormalities. However, it is likely that chromosome 15 is the acrocentric chromosome most likely to be influenced by the $15 \mathrm{p}$ polymorphism and apart from the acrocentric marker chromosome group of patients in which there were 45 marker 15 chromosomes, there was only one trisomy 15 patients and only five Robertsonian translocations involving chromosome 15. Virtually all our patients were Caucasians and it would be interesting to determine whether the $15 \mathrm{p} 11$ polymorphism is present in other populations and, if so, if the frequency is similar to that seen in our population.

\section{Acknowledgements}

We thank Sarah Beal and the Molecular Cytogenetics team for the preparation of the probes used in this study. 


\section{References}

1 Spowart G: Reassessment of presumed Y/22 and Y/15 translocations in man using a new technique. Cytogenet Cell Genet 1979; 23: $90-94$

2 Babu A, Macera MJ, Verma RS: Intensity heteromorphisms of human chromosome $15 \mathrm{p}$ by DA/DAPI technique. Hum Genet 1986; 73: 298-300.

3 Perez-Castillo A, Martin-Lucas MA, Abrisqueta JA: Evidence of the lack of specificity of the DA/DAPI technique. Cytogenet Cell Genet 1987; 45: 62-64.

4 Higgins MJ, Wang HS, Shtromas I et al: Organization of a repetitive human $1.8 \mathrm{~kb} \mathrm{KpnI}$ sequence localized in the heterochromatin of chromosome 15. Chromosoma 1985; 93: 77-86.

5 Wilkinson TA, Crolla JA: Molecular cytogenetic characterization of three familial cases of satellited Y chromosomes. Hum Genet 1993; 91: 389-391.

6 Shim SH, Pan A, Huang XL et al: FISH variants with D15Z1. J Assoc Genet Technol 2003; 29: 146-151.

7 Smeets DFCM, Merkx GFM, Hopman AHM: Frequent occurrence of translocations of the short arm of chromosome 15 to other D-group chromosomes. Hum Genet 1991; 87: 45-48.
8 Stergianou K, Gould CP, Waters JJ, Hultén M: High population incidence of the $15 \mathrm{p}$ marker D15Z1 mapping to the short arm of one homologue 14. Hum Genet 1992; 88: 364

9 Stergianou K, Gould CP, Waters JJ, Hultén M: A DA/DAPI positive human $14 \mathrm{p}$ heteromorphism defined by fluorescence in-situ hybridisation using chromosome 15-specific probes D15Z1(satellite III) and p-TRA-25 (alphoid). Hereditas 1993; 119: $105-110$.

10 Cockwell AE, Jacob PA, Beal SJ, Crolla JA: A study of cryptic terminal chromosome rearrangements in recurrent miscarriage couples detects unsuspected acrocentric pericentromeric abnormalities. Hum Genet 2003; 112: 298-302.

11 Sambrook J, Russell D (eds): Molecular cloning: a laboratory manual, (3rd edition) Cold Spring Harbor, New York: Cold Spring Harbor Laboratory Press, 2001

12 Pinkel D, Landegant J, Collins C et al: Fluorescence in situ hybridization with human chromosome-specific libraries: Detection of trisomy 21 and translocations of chromosome 4. Proc Nat Acad Sci USA 1988; 85: 9138-9142.

13 Redon R, Ishikawa S, Fitch KR et al: Global variation in copy number in the human genome. Nature 2006; 444: 444-454. 